\title{
Magnetic complexity, fragmentation, particle acceleration and radio emission from the Sun
}

\author{
Loukas Vlahos \\ Department of Physics, \\ University of Thessaloniki, \\ 54124 Thessaloniki, Greece. \\ vlahos@astro.auth.gr
}

\begin{abstract}
The most popular flare model used to explain the energy release, particle acceleration and radio emission is based on the following assumptions: (1) The formation of a current sheet above a magnetic loop, (2) The stochastic acceleration of particles in the current sheet at the helmet of the loop, (3) the transport and trapping of particles inside the flaring loop. We review the observational consequences of the above model and try to generalize by putting forward a new suggestion, namely assuming that a complex active region driven by the photospheric motions forms naturally a large number of stochastic current sheets that accelerate particles, which in turn can be trapped or move along complex field line structures. The emphasis will be placed on the efficiency and the observational tests of the different models proposed for a flare
\end{abstract}

\section{Introduction}

Radio emission from solar active regions during flares is closely related with two factors, (a) the rate of electron acceleration before, during and after the impulsive phase of the flare, and (b) the topology of the magnetic field in the active region hosting the flare. None of the main "actors" responsible for the characteristics of the radio emission is well known and this makes the direct modeling and interpretation of the radio observations extremely difficult.

High energy particles are also responsible for hard X-rays and $\gamma$-ray bursts emitted from the same active region and are less dependent on the details of the magnetic field topology. The combined analysis of the high energy emission (including Hard X-rays, $\gamma$-rays and radio) from the same event is an extremely valuable tool for our understanding of the physical processes behind the flares. Unfortunately, the events covered simultaneously in all wavelengths are only a few, and no conclusive results can be drawn for the source of energetic particles or the topology of the magnetic fields.

We can then conclude from the above that the direct reconstruction of the velocity distribution and the magnetic topology from the data is not possible, which has led many researchers to very simplistic models (Maxwellian with power law tails, simple magnetic loops, single current sheets, etc). The alternative road is to estimate the high energy emission from the proposed flare model (direct modeling) and then compare the results of the model with the 
available data. This road seems more straightforward, but there is a fundamental obstacle, the scales of the physical processes involved in the formation of the unstable magnetic topology responsible for the flare are very different from those responsible for the dissipation of the magnetic energy and the acceleration of high energy particles.

MHD models can follow successfully the large scale evolution of the magnetic field, but the dissipation of magnetic energy (responsible for flares) is not necessarily an MHD processes (resistivity plays a crucial role). The acceleration of particles is a kinetic phenomenon appearing on all scales. Therefore, all attempts made so far to explain the flare and the CME inside the framework of the MHD theory have failed to explain the high energy phenomena.

In the past, high energy particles (energies above $25 \mathrm{keV}$ ) were assumed to carry a small percentage of the energy released in explosive phenomena. Recent estimates show that this is not true and more than $40 \%$ of the energy released in solar flares is going to high energy particles $[1,2,3,4,5]$. This leads us to the conclusion that kinetic phenomena play a crucial role in the understanding of solar flares.

Hybrid codes, following both, the evolution of the MHD and kinetic aspects of a flare on all scales (from meters to thousand of kilometers) are not feasible today and the detailed modeling of solar flares remains an open problem for the future generation of computers. We then conclude that, since both the reverse and forward modeling are not possible today, we will go ahead with simple scenarios (cartoons) for the processes which we believe to be active during flares and follow their implications.

Two broad classes of flare models are widely used today: (a) The breakup type of models, representing mainly the flares which show close association with large scale events leading to CMEs $[6,7,8]$, and (b) the loop models, which explains better the compact and relatively small flares which are not associated with CMEs. The splitting of the physical processes in categories is a useful tool for detailed studies, but it may lead us to wrong conclusions when the magnetic topologies, where the flares start, are extremely complex.

The mechanisms for the acceleration of particles and their transport is different in the two models. The expected radiation signatures are also very different and it is worth reviewing briefly the main features.

Our goal in this review is to present the current status of the energy release processes for solar flares and the associated acceleration mechanisms and to sketch the expected radio emission. Moreover we introduce a third type of flare model, which is based on the complexity of the magnetic topologies and the fragmentation of the magnetic energy release, which is associated with the turbulent photospheric motions and which results in complex magnetic fields and the formation of many current sheets of all scales.

In section 2, the main "traditional" models (break up and loops) for the energy release and the expected acceleration of particles will be sketched. In 
section 3 , the energy release and the acceleration of particles in more realistic complex magnetic topologies will be analyzed and in section 4 the expected radio emission from all three types of models will be discussed briefly. Our results will be summarized in section 5 .

\section{Classical models for energy release and particle acceleration}

\subsection{The break-up model for flares/CME}

A large variety of models demonstrates the connection between the flare and the CME $[9,10,11,12,13,14,15]$. All the above models start from simple magnetic topologies (2D or $3 \mathrm{D}$ ) which are driven to instability by the shear along or perpendicular to a neutral line or by emerging magnetic flux. The schematic view of the models emerging from the proposed scenarios and corresponding simulations are shown in Fig. 1. There are several variants of the break-up model, according to the details of the initial magnetic field and the photospheric motions, these details are though beyond the scope of this article (see [16]).

The cartoon presented in Fig. 1a shows that the high energy particles are covering a small portion (labeled with red) of the volume covered by the unstable structure. There are several acceleration regions in this model: (1) the current sheet, (2) the turbulent outflows, (3) the slow and fast shocks. In Fig. 1b, the emphasis is given on the presence of several shocks surrounding the long and thin current sheet above the closed magnetic topology.

We could point out several weak points of the break up model, we will though focus our attention on the so called "number problem". It is well known that during a flare the required rate of particles accelerated is $10^{37}$ particles/sec, so for a flare lasting 100 secs more than $10^{39}$ particles will be accelerated. Translating this number to coronal conditions (mean density $10^{9}-10^{8}$ particles $/ \mathrm{cm}^{3}$ ), we conclude that the current sheet and the surrounding parts (fast jets, shocks etc) (see Fig. 1a) should cover a volume comparable to $10^{28} \mathrm{~cm}^{3}$. Assuming that the thickness of the current sheet is several $\sim 10 \mathrm{~km}$ we can reach the conclusion that the current sheet should be huge $\left(10^{11} \mathrm{~cm} \times 10^{11} \mathrm{~cm}\right)$ and should remain stable for $100 \mathrm{~s}$ of secs. The plasma inside this volume should be replenished and accelerated to high energies with extraordinary efficiency. We believe that it is hard to prove that this huge structure will remain stable and active for so long inside an unstable magnetic topology. The break up of the current sheet and the formation of several fragments will be a natural consequence [17].

The 3D evolution of a simple bipolar photospheric magnetic field leads also to the break up model, but the magnetic topology is extremely complex [15]. The formation of a large number of stressed magnetic field lines (see Fig. 2) with numerous current filaments and fragments is apparent and can 


\section{CME/Flare Loop Structures}
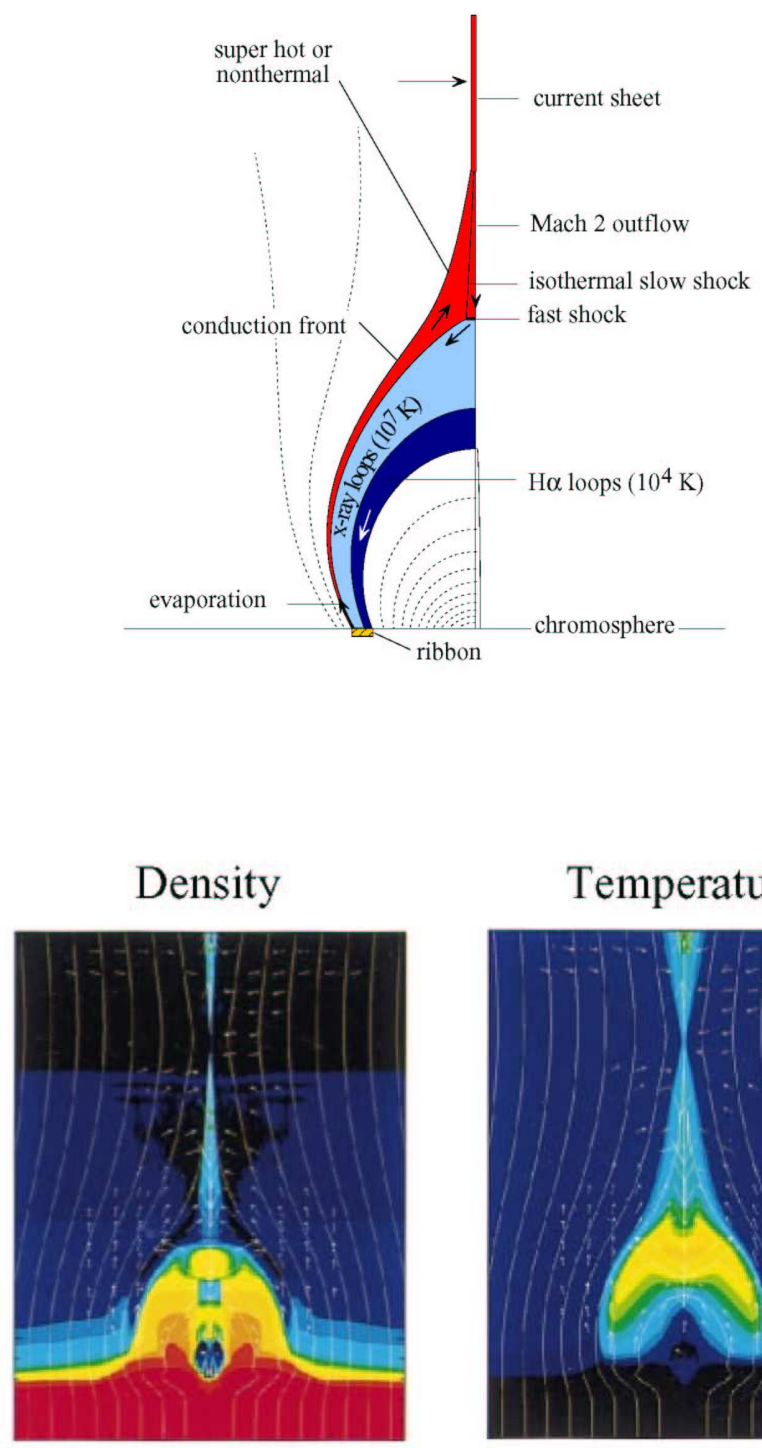

\section{Temperature}

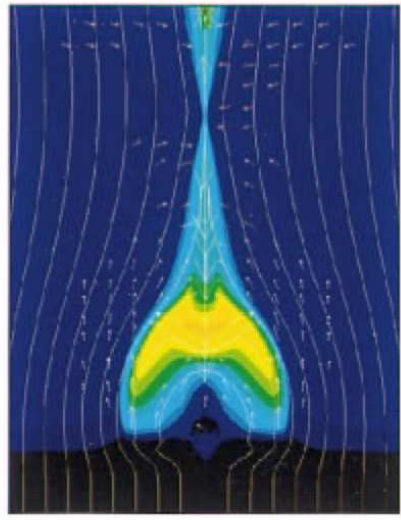

Fig. 1. The break up model. (a) The schematic representation [11], (b) A 2-D simulation [12]. 
be the answer to the high energy emission observed. The simple magnetic topology for the current sheet, presented earlier and the associated simple accelerators (Direct E-field, constant flows and shocks) are replaced with much more complex topologies, which host a variety of accelerators in the $3 \mathrm{D}$ configurations.

The most prominent accelerators in the stressed 3D topologies mentioned above are the stochastic electric fields due to the sheared field lines. The expected electric fields are

$$
\boldsymbol{E}(\boldsymbol{r}, t)=-\boldsymbol{V}(\boldsymbol{r}, t) \times \boldsymbol{B}(\boldsymbol{r}, t)+\eta \boldsymbol{J}(\boldsymbol{r}, t)
$$

where $\boldsymbol{V}(\boldsymbol{r}, t)$ is the plasma velocity, $\eta$ is the resistivity, $\boldsymbol{B}(\boldsymbol{r}, t)$ the magnetic field and $\boldsymbol{J}(\boldsymbol{r}, t) \sim \nabla \times \boldsymbol{B}(\boldsymbol{r}, t)$ the current. In the 3D representation of the break up model the monolithic current sheet disappears and new, more advanced and interesting models for particle acceleration appear. We will come back to these models in the next section.

\subsection{The loop model}

Nordlund and Galsgaard [18] solved numerically the non-ideal MHD equations to follow the evolution of photospheric stresses on a simple magnetic loop. The magnetic field initially was assumed to be uniform and anchored in the photosphere. The boundary velocity consists of a sinusoidal shear with a wave length equal to the length of the boundary. The orientation of the shear, the phase, the velocity amplitude and the duration of the individual driving events are random, with various limitations on the parameters [19].

As the boundaries of the loop are stressed by the randomly changing boundary flows, the loop is stressed into a state where numerous Unstable Current Sheets (UCS) are formed and distributed along the length of the loop (see Fig. 3).

Turkmani et al. [20] used the the above model to study its efficiency to accelerate particles. A snapshot of the coronal magnetic field was used. The 3D structure of the electric field was estimated with the use of Eq. 1 (see Fig. 4). The scattered electric field accelerates ions and electrons in very short times $(\leq 0.1 \mathrm{sec})$. The electrons and ions are stochastically accelerated forming energy distributions similar to the ones needed to reproduce the observations. So far we have analyzed the random formation of stresses inside the loop. The loop is also disturbed by Alfven waves propagating along the magnetic field. It is well known that weak MHD turbulence is a very efficient accelerator [21]. Diffusive acceleration of particles by MHD waves was contrasted and compared to direct E-fields and shocks formed by large scale current sheets in the break-up model. Several recent articles showed that the non linear evolution of the MHD waves form small scale structures, which act also as shocks or UCS [23, 22, 24].

Arzner and Vlahos [23] discuss the efficiency of particle acceleration in the presence of isotropic MHD turbulence with anomalous resistivity as a proxy 
3D Flux Rope Simulation

Roussev et al. (2003)

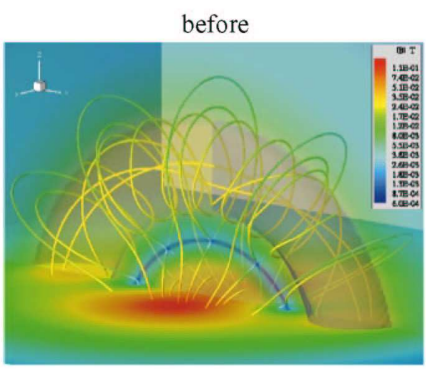

after
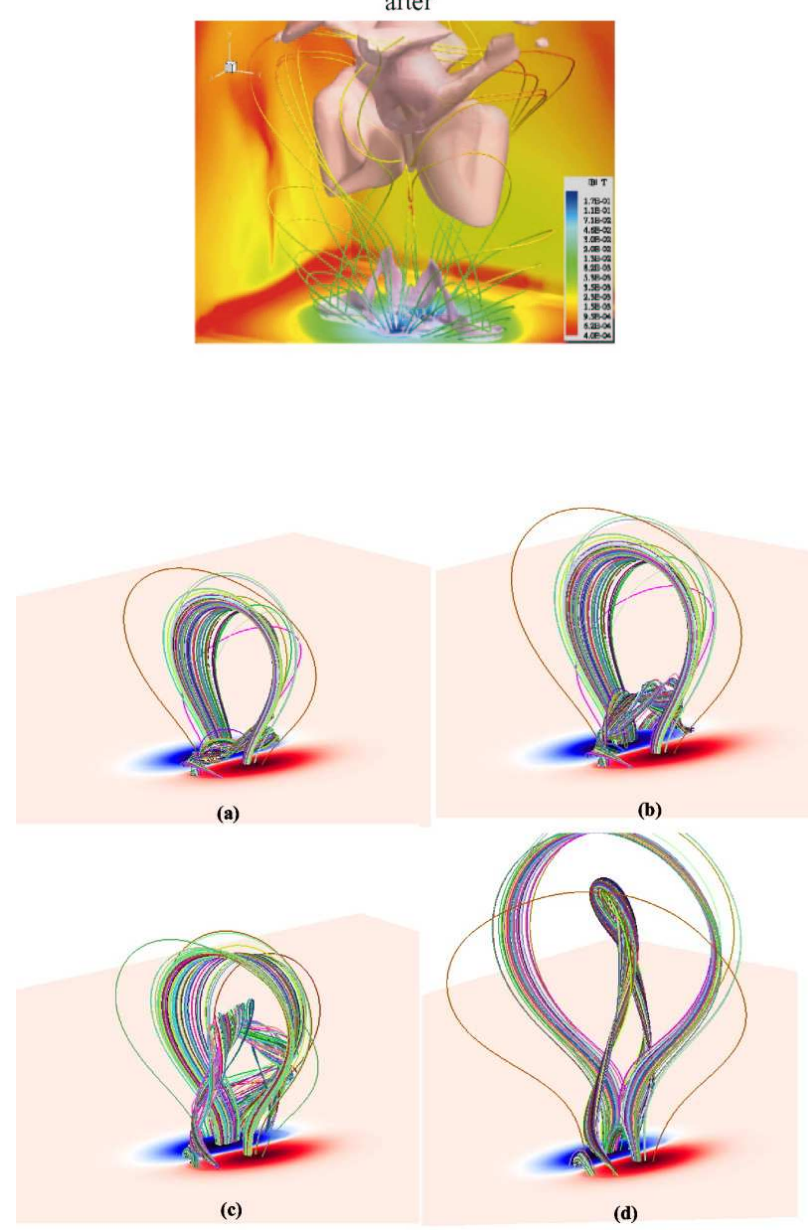

Fig. 2. Using the $3 \mathrm{D}$ MHD equations, even by starting from a simple magnetic geometry, the loop is led to a break up state with a very complex magnetic topology. The formation of numerous current sheets on all scales is apparent $[14,15]$. 


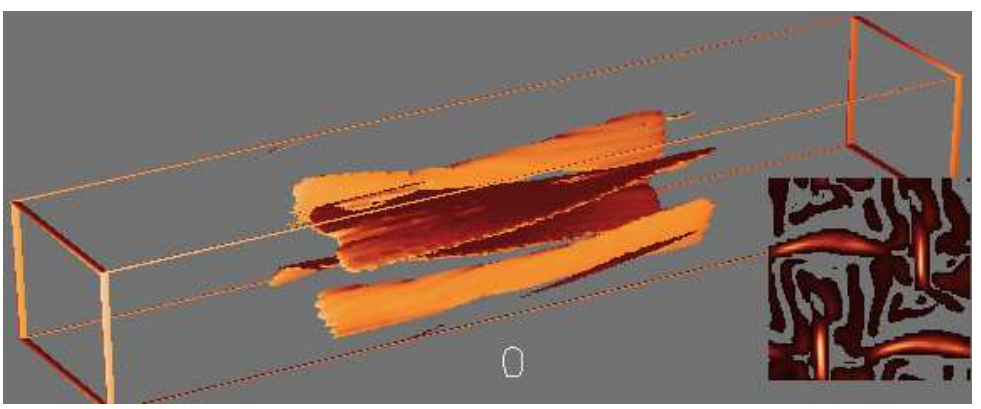

Fig. 3. The loop is stressed by random photospheric flows and is led to a state where numerous current sheets are present. A vertical cross section through the middle of the loop shows the formation of current sheets [18]

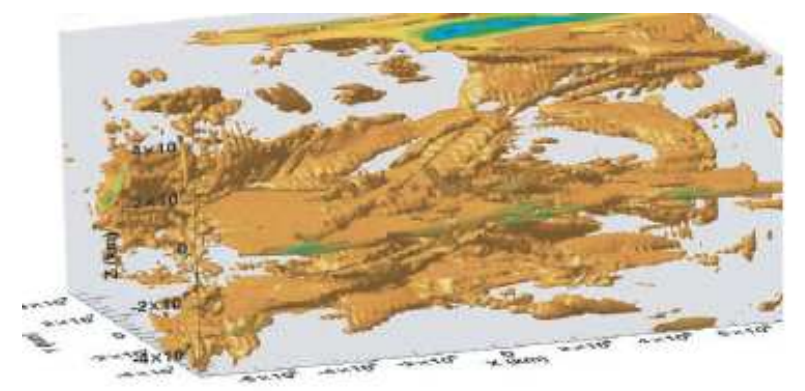

Fig. 4. The resistive electric field within the coronal loop, as calculated by the MHD model [20]

for the solar corona. The model for the MHD turbulence was relatively simple. They assume that the vector potential $\boldsymbol{A}$ was a superposition of Alfven waves propagating along the external magnetic field $\boldsymbol{B}_{0}$

$$
\boldsymbol{A}=\sum_{k} \boldsymbol{a}(\mathbf{k}) \cos \left(\mathbf{k} \cdot \mathbf{x}-\omega(\boldsymbol{k}) t-\phi_{k}\right)
$$

in axial gauge, $\mathbf{a}(\mathbf{k}) \cdot \mathbf{v}_{A}=0$ and with the dispersion relation $\omega(\boldsymbol{k})=\boldsymbol{v}_{A} \cdot \boldsymbol{k}$, which is an exact solution of the induction equation with constant velocity $\boldsymbol{v}_{A}$. The $\boldsymbol{A}(\boldsymbol{x}, \mathrm{t})$ is taken as Gaussian with random phases $\phi_{k}$ and (independent) Gaussian amplitudes $\mathbf{a}(\mathbf{k})$, with zero mean and variance.

They analyze the evolution of a collisonless test particle in evolved homogeneous MHD turbulence with electromagnetic fields

$$
\begin{gathered}
\boldsymbol{B}=\nabla \times \boldsymbol{A} \\
\boldsymbol{E}=-\partial_{t} \boldsymbol{A}+\eta(J) \boldsymbol{J}
\end{gathered}
$$

where $\mu_{0} \boldsymbol{J}=\nabla \times \boldsymbol{B}$ and the resistivity switches on to anomalously high values when the current exceeds a critical value 


$$
\eta(J)=\eta_{0} \theta\left(|J|-J_{c}\right)
$$

where $\theta(x)$ is the step function. The wave vector represents the random fluctuations along the extremal magnetic field. The formation of UCS inside the $3 \mathrm{D}$ topology of the magnetic field is a consequence of the non linear interaction of MHD waves with the plasma (see Fig. 5a).
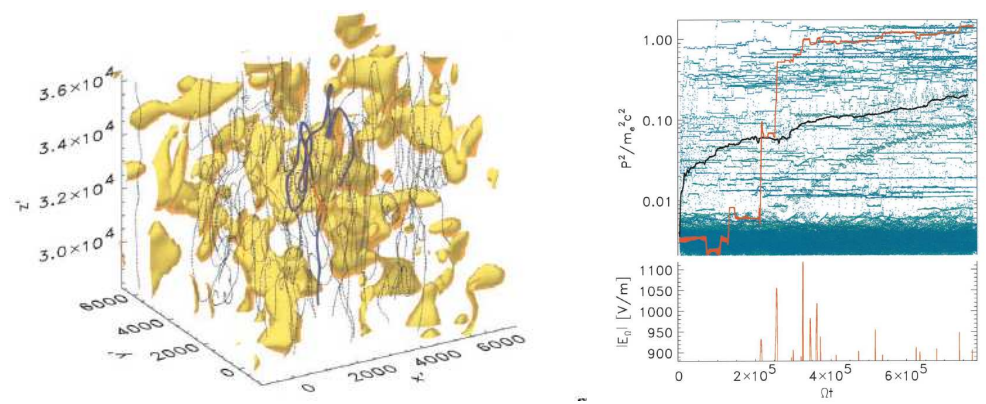

Fig. 5. (a) Location of the UCS. (b) Evolution of the electron momentum. Samples of trajectories show that the particles perform random walks and a few particles undergo very fast acceleration. All particles visit a sample of UCS [23].

Particles crossing the localized UCS will experience a sudden acceleration (or deceleration) ( See Fig. 5b). These jumps are random and their characteristics are shown to be beyond the quasilinear analysis described by the standard Fokker-Planck equation [25]. We can then conclude that for high amplitude MHD waves the simple division between waves, shocks and large scale UCS is lost, and it is replaced with particle acceleration in a mixture of waves, UCS and shocks.

\section{Energy release and particle acceleration in complex magnetic topologies}

The initial magnetic topologies used so far in all the models discussed above were relatively simple bi-polar regions. The initially stable magnetic topologies were forced to instability by the continuous or random stressing from the photospheric motions. The evolution of the large scale instability led to the formation of a complex, fragmented structure.

The next level of complexity is to use a realistic magnetic field topology born out from the linear and non linear force free extrapolation of the observed photospheric magnetic fields.

Vlahos and Georgoulis [26] use as the starting point of their analysis the magnetograms from a non flaring active region. Using the simplest possible method for the force free extrapolation [27], they determined the 3D magnetic 
field topology inside the AR. The real magnetic topologies are even more complex than the magnetic fields predicted by the linear force free state, but for the statistical analysis presented in their article the linear force free extrapolation is probably suitable. Using simple criteria for the potentially unstable currents, e.g the angular difference between two adjacent magnetic field vectors, $\boldsymbol{B}_{1}$ and $\boldsymbol{B}_{2}$ to exceed a certain value, since the steep magnetic field gradients favor magnetic reconnection in 3D magnetic topologies [28], they were able to define the location of the UCS. They concluded that AR form naturally UCS even during their formation stage (Fig. 6). The free
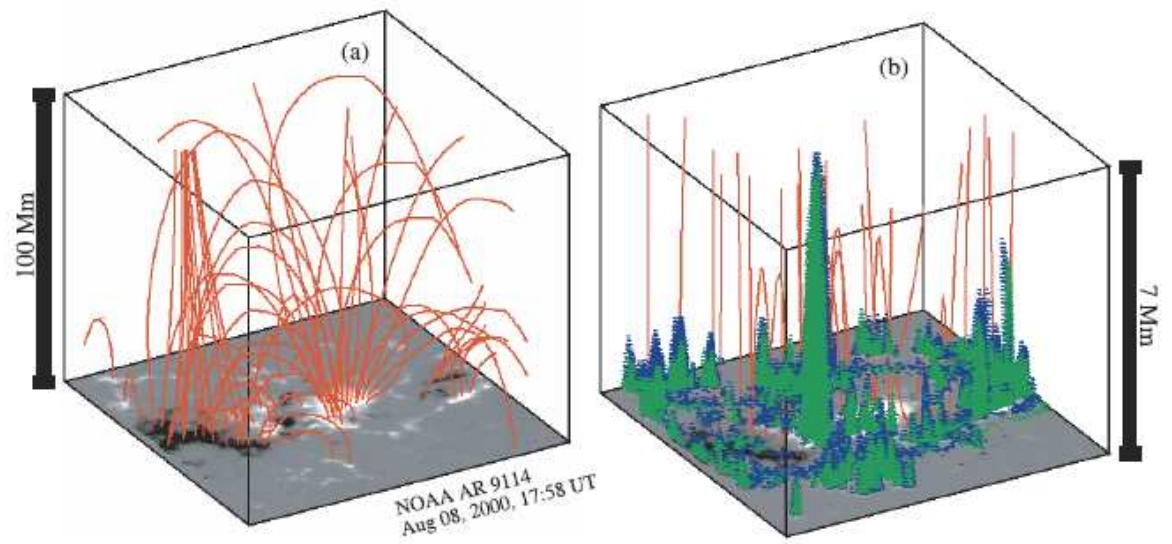

Fig. 6. (a) Linear force free field extrapolation in NOAA AR 9114, (b) Lower part of the AR atmosphere. Shown are the magnetic field lines (red) with the identified discontinuities for critical angle $10^{\circ}$ [26].

energy available in these unstable volumes follows a power law distribution with a well defined exponent (Fig. 7) [26]. We can then conclude that AR store energy in many unstable spots forming UCS of all sizes. The UCS are fragmented and distributed inside the global 3D structure.

The only approach which is capable to capture the full extent of the interplay of highly localized dissipation in a well-behaved large scale topology ("sporadic flaring") is based on a special class of models which use the concept of Self-Organized Criticality [29]. The main idea is that active regions evolve by the continuous addition of new or the change of existing magnetic flux on an existing large scale magnetic topology, until at some point(s) inside the structure magnetic discontinuities are formed and the currents associated with them reach a threshold. This causes a fast rearrangement of the local magnetic topology and the release of the excess magnetic energy at the unstable point(s). This rearrangement may in turn cause the lack of stability in the neighborhood, and so forth, leading to the appearance of flares (avalanches) 


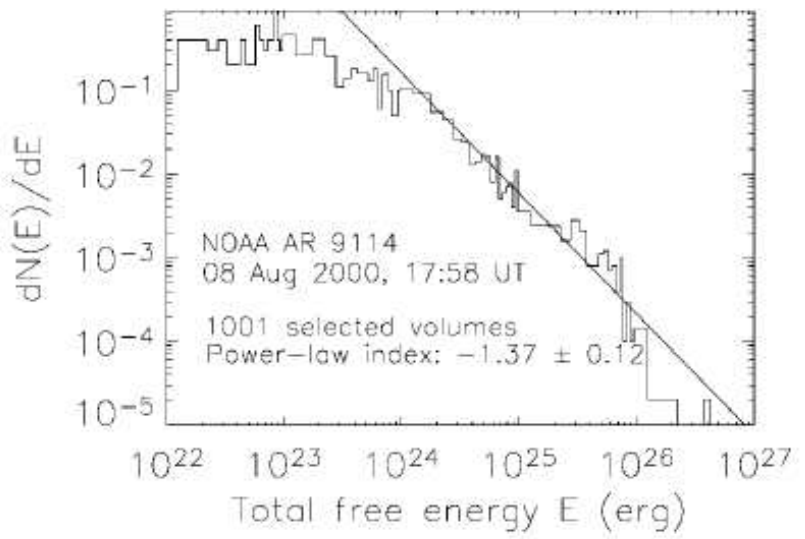

Fig. 7. Typical distribution function of the total free energy in the selected volume, on using a critical angle $14^{\circ}[26]$.

of all sizes that follow a well defined statistical law [30, 31, 32], which agrees remarkably well with the observed flare statistics [33]. We can then conclude, after many years of studies, that a possible model for the dynamic evolution of the AR is the following: The 3D magnetic field is stressed from the photospheric motions, forms continuously UCS which relax, re-arranging the local magnetic field and causing flares of all sizes (Fig. 8).

The acceleration of particles inside a complex active region being in Self Organized Critical state has been analyzed in numerous articles $[35,37,36]$.

We now pose a new question: Can the UCS become the local nodes for a large evolving network and accelerate stochastically electrons and ions? In this case the accelerator is not located in a single volume but it is distributed along the trajectory of the particle (see Fig. 9).

Vlahos, Isliker and Lepreti [37] study the statistical properties of an ensemble of isolated UCSs, and investigate the statistics of the energy gain when an entire distribution of particles moves through spatially distributed UCSs, all particles having random initial conditions. This question belongs to the field of MHD in combination with kinetic plasma physics (in what refers to anomalous resistivity). Also needed is an understanding of the spatial organization of an ensemble of co-existing UCSs and of their connectivity and evolution. A first hint to how the UCS might be organized spatially comes from the cited inquiries of SOC models, which are in favor of a global fractal structure with dimension around 1.8. The problem actually concerns the nature of 3-D, large scale, magnetized MHD turbulence, and it involves theory as well as observations.

With the concrete specifications of the random walk to the solar flare problem they made, they were able to achieve HXR spectra which are com- 


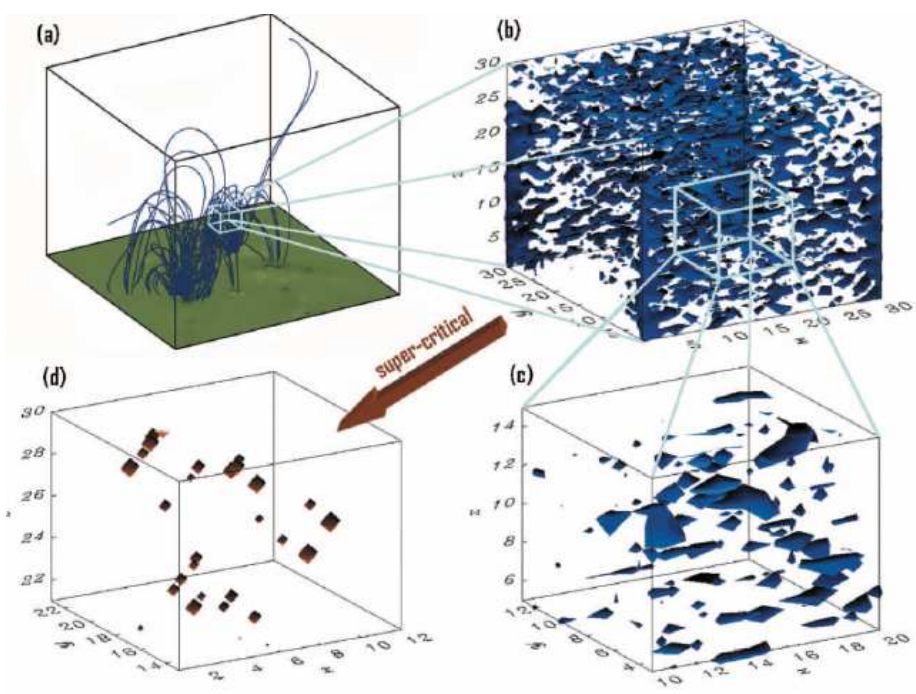

Fig. 8. (a) Simulated magnetogram of a photospheric AR and force free magnetic field lines, extrapolated into the corona (see [34]), (b) Subcritical current isosurfaces in space, as yield by the X-CA model [32], a particular SOC model, which models a subvolume of a coronal AR. (c) Same as (b), but zoomed. (d) Temporal snapshot of the X-CA model during a flare, showing the spatial distribution of the UCS inside the complex AR [37].

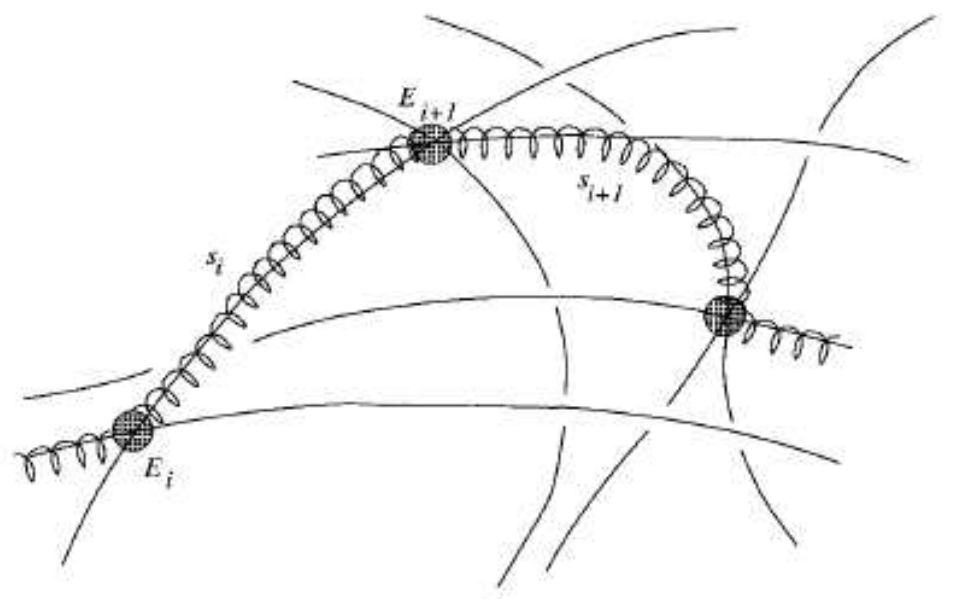

Fig. 9. A particle (spiraling line) follows the magnetic filed lines (solid lines), travels freely a distance $s_{i}$ until it enters a UCS (filled circle) where it is accelerated by the associated effective DC field $E_{i+1}$. After the acceleration event the particle again moves freely till the particle meets the next acceleration event [37]. 
patible with the observations. Important is that the model naturally leads to heating of the plasma, or, more precisely, it creates a heated population in the plasma. This heated population can be expected to heat the entire background plasma through collisional interactions on collisional time-scales, explaining in this way the observed delay between the thermal soft X-ray and the non-thermal hard X-ray emission.

\section{Radio emission from simple and complex magnetic topologies}

We are now ready to pose a very important question: Is it possible to identify the concrete radio signatures from the complexity of the magnetic field lines, the energy release and particle acceleration? The exact modeling of the radio emission from the structures presented above is still lacking but we can make several preliminary comments, hoping that both the new development in the theory and more importantly the new data expected from the Frequency Agile Solar Radiotelescope (FASR) [38] will give a new input to the analysis presented in this review. Let us discuss separately the well known parts of the flare related radio emission, staring from the microwave bursts.

\section{Microwave bursts}

Microwave bursts are currently interpreted as the signature of mildly relativistic electrons trapped inside a magnetic loop [39, 40]. Acceleration of electrons and ions inside the loop can be a natural explanation for several well known high energy emissions: (1) The precipitating, mildly relativistic electrons produce the hard X-ray bursts forming the well known foot point emission, (2) the trapped mildly relativistic electrons produce the microwave bursts, forming large scale sources at the "loop top", (3) the precipitating relativistic electrons and ions are responsible for the $\gamma$-ray bursts.

Trapped and precipitating populations will also be present in more complex magnetic topologies. The fragmented energy release inside the loop will accelerate the electrons to almost relativistic energies [20]. The accelerated particles will cover large distances (thousands of kilometers) in relatively short time scales (fraction of a second), therefore microwave emission is not fragmented because the particles fill in a short time large parts of space, having moved away from the acceleration regions.

Radio instruments able to map the flaring region on a fast time scale (sub sec) can probably record, at the start of the flare, many small sources (dcm spikes?), but eventually this will give away to a large and almost uniform source at later times.

The simple version of the break-up model will easily provide the precipitating particles and explain the Hard X-rays and $\gamma$-ray bursts but the 
trapping of particles inside the closed loop below the helmet is difficult to explain (cross field diffusion is rather difficult for these energies). Therefore we should expect a long delay (tens of minutes) between microwave bursts and Hard X-rays (such delays have not been recorded). The 3D analog of the break-up model leaves more room for precipitating and trapped particle with much less delay. Nobody has attempted so far to follow particles in a 3D magnetic topology resulting from the break-up model and hosting many reconnection sites.

We can then conclude that hard $\mathrm{X}$ rays, microwaves and $\gamma$-rays can easily be accommodated from the third type of flare models, which incorporates the fragmented energy release. We predict that the new generation of radio instruments will record many isolated microwaves sources at the start of the flare.

\section{dcm spikes, Type III bursts}

The simple versions (assuming monolithic current sheets) of both flare models (loop model and the break-up) cannot account for the above bursts. Type IIIs appear usually in groups (isolated type IIIs are rare) at the rising phase of a flare.

These bursts can be explained from the fragmentation of the energy release in realistic magnetic topologies reconstructed from the extrapolation of the observed photosheric magnetic fields. Both types of magnetic field lines are present (closed and open, see Fig. 6). Therefore complex magnetic topologies and fragmentation are probably the explanation for the groups of type IIIs and dcm spikes (see more in [41]).

A stochastic model for type III bursts was introduced and compared with observations [42]. In this model the AR is assumed inhomogeneous with a very large number of fragmented energy release regions (UCSs) connected to magnetic fibers. At the base of the magnetic fibers, random energy release events take place, in the course of which electrons are accelerated, travel along the fibers and eventually undergo bump-on-tail instability. Their main conclusion was that the observations are comparable with this model (see Fig. 10).

\section{Type II bursts}

The break up model can easily account for the type II emission. It predicts several shocks, traveling in all directions, but type II bursts (forward and reverse) are not so common during flares. We can then ask: Why are type II bursts not always part of the flare/CME complex? The loop model on the other hand is not an efficient source of type II bursts. 

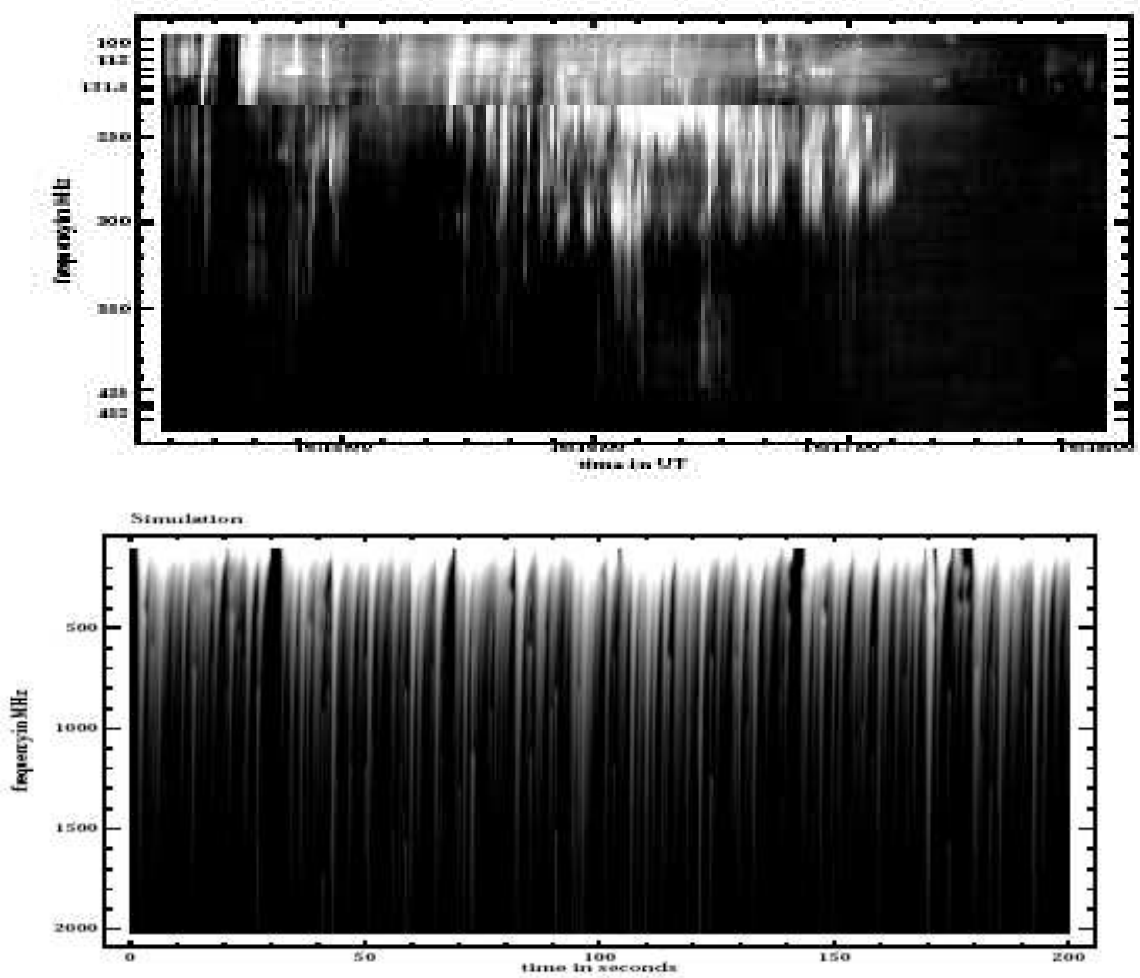

Fig. 10. (a) Spectrogram of type III event on 1980/06/27, 16:14:18 UT (time resolution $0.1 \mathrm{sec}$, shown duration 200 secs (b)200 secs of a spectogram generated by the model with a time resolution $0.02 \mathrm{sec}$ and frequency resolution $55.9 \mathrm{MHz}$. $[42]$.

\section{Emission before and after the flare}

The 3D version of the break up model can explain the pre-event radio emission as an expression of the build up of stresses leading to the instability, but it has difficulties to explain the long lasting (sometimes lasting for days) emission after the flare. The compact flare model can explain both since the loop is constantly under stress and the flare is a stronger explosion out of a series of explosions of all sizes. The fragmented complex loops model can explain these emissions much more easily. The realistic magnetic topologies easily predict both types of activity (pre flare and post flare). It is a challenge for the new radio instruments to investigate deeper this part of the flare problem.

\section{Type I and type IV bursts}

Type I and type IV continuum bursts can be the result of fragmentation of the energy release in large scale coronal magnetic complexes. The fragmented 
acceleration sources are responsible for the type I bursts. We propose that type I bursts are closely connected with the UCSs in the upper corona. Particle acceleration from the ensemble of the UCSs and subsequent trapping are responsible for the type IV continuum. The type I/IV bursts are analogous to the dcm spikes/ microwave bursts for the upper corona. The fundamental difference between the two types of bursts is that the dcm spikes/microwaves are powered by a flare and the type I/ IV are related with the micro flares appearing in the upper corona.

\section{Summary}

We have suggested in this review that flare models can be split into three very broad classes. The break-up model is responsible for the flares associated with CMEs and the loop model is connected with the compact flares. The complex model is an extension and generalization of the two classical models.

In 3D simulations of the break up model and of a randomly stressed loop the initial simple magnetic topology is forced to create many reconnection sites, and large scale current sheets collapse into many fragments.

Acceleration of particles is much simpler in an environment of fragmented energy release since the presence of stochastic E-fields, appearing in stressed magnetic topologies, naturally produces many UCS which collectively act to accelerate particles.

The next step in the development of realistic models is to consider the loop model and the break-up model in magnetic topologies born out from the observed photospheric magnetic topologies (using linear or non linear force free extrapolation as the basic tool). In these cases the sharp division between the break-up and the loop flare model starts to disappear and a third type of model based on the fragmentation of energy release emerges, as shown here.

Current observations give only partial support for the break-up or the loop flare model. There are observations fitting naturally in the one or the other model and others which are hard to fit in any. We believe that the extrapolated magnetic fields, stressed by turbulent photospheric motions will create a mixture of closed and open field lines populated by randomly placed E-fields and they will be able to model the known observations. These topologies have the following characteristics

- They are efficient accelerators;

- They have a mixture of open and closed magnetic filed lines

- They can explain most types of bursts.

We believe that soon the next generation of flare models will emerge, where the current sheets will be hosted in a mixture of open and closed field lines. Forward modeling of the well known bursts inside these topologies will be an important diagnostic tool that will allow comparison to results from the radio instruments which are currently under development. 
Acknowledgements: This work was supported in part by the Research Training Network (RTN) 'Theory, Observation and Simulation of Turbulence in Space Plasmas', funded by the European Commission (contract No. HPRN-eT-2001-00310). I would like to thank my colleagues, Drs Heinz Isliker, Manolis Georgoulis, Anastasios Anastasiadis, Kaspar Arzner, Rim Turkmani, Klaus Galsgaard, Bernhard Kliem and Prof. Peter Cargill for discussing and developing together several ideas presented in this review.

\section{References}

1. R.B. Lin, H.S. Hudson: Sol. Phys. 50, 153, (1976)

2. B. R. Dennis, R.A. Schwartz: Sol. Phys. 121, 75, (1989)

3. A.O. Benz et al.: Sol. Phys. 153, 33, (1994)

4. P. Saint-Hilaire, A.O. Benz: Sol. Phys. 210, 287, (2002)

5. G.D. Hollman et al., ApJ., 595, L97, (2003)

6. E. R. Priest, T.G. Forbes: Astr. Astroph. Rev, 10,313

7. B.C. Low: J. Geoph. Res., 106, 25141, 2001

8. J.A. Klimchuk: in "Space Weather", P. Song, H.J. Singer and G.L.Siscoe (eds), AGU Geophysical Monograph, 125, 143, (2001)

9. R.A. Kopp, G.W. Pneuman: Solar Phys. 216, 123 (1977)

10. J. Heyvaerts, E.R. Priest, D. Rust: ApJ, 50, 85 (1976)

11. T.G. Forbes, E.R. Priest: ApJ, 446, 377 (1995)

12. T. Yokoyama, K. Shibata: ApJ, 549, 1160 (2001)

13. S. Antiochos, C. R. DeVore, J. A. Klimchuk: ApJ, 510, 485 (1999)

14. I.I. Rousev et al: ApJL, 588, L45 (2003)

15. T. Amari, J. F. Luciani, J.J. Aly, Z. Mikic and J. Linker: ApJ, 595, 1231 (2003)

16. M. Aschwanden: Physics of the solar corona, (Springer, Berlin Heidelberg New York 2004).

17. B. Kliem, M. Karlisky, A.O. Benz: Astr. Astrophy., 360, 715 (2000)

18. A. Nordlund, K. Galsgaard, : Tech. Rept., Astr. Obs., Copenhagen Univ. (1997)

19. K. Galsgaard, A. Nordlund: J. Geoph. Res.,101, 13445 (1996)

20. R. Turkmani et al.: ApJ., 620, L59 (2005)

21. J. A. Miller et al: Rev. Geoph. Res.,102, 14631 (1977)

22. P. Dmitruk et al: ApJ,617, 667 (2004)

23. K. Arzner, L. Vlahos: ApJL, 605, L69 (2004)

24. S. Moriyasu et al.: ApJL, 601, L107 (2004)

25. K. Arzner, L. Vlahos, B. Knaepen and N. Denewet: Notes in Computer Sciences, in press (2005)

26. L. Vlahos, M. Georgoulis: ApJL, 603, L61 (2004)

27. C. E. Alissandrakis: Astr. Astroph., 100, 197 (1981)

28. E. Priest, G. Horning, D.I. Pontin: J. Geoph. Res., 108(A7), 1285 (2003)

29. Bak, P., Tang, C., Wiesenfeld, K., 1987, Phys. Rev. Lett. 59, 381.

30. E.T. Lu, RJ. Hamilton: ApJ, 380, L89 (1991)

31. L. Vlahos, M. Georgoulis, R. Kluving and P. Paschos: AA,299, 897 (1995)

32. H. Isliker, A. Anastasiades, L. Vlahos: AA,377, 1068 (2001) 
33. Crosby,N.B., Aschwanden, N.J. and Dennis, B.R., 1993, Sol. Phys. 143, 275 (1993)

34. T. Fragos, E. Rantsiou, L. Vlahos: Astr. Astroph., 420, 719 (2004)

35. A. Anastasiadis, M. Georgoulis, L. Vlahos.: ApJ, 489, 367 (1997)

36. A. Anastasiadis, C. Gontikakis, N. Vilmer, L. Vlahos.: AA, 603, 422323 (2004)

37. L. Vlahos, H. Isliker, F. Lepreti: ApJ, 608, 540 (2004)

38. T. Bastian: Ad Space Res., 32, 2705 (2003)

39. Kundu, M.R., Vlahos, L.: Space Sci Rev., 32, 405

40. T. Bastian :Proc-2000-Murdin,Vol. 3, 2553 (2000)

41. A. Benz: Solar and Space Weather Radiophysics, (eds D.E. Gary and C.U. Keller) Kluwer Academic Press, 203-221 (2004)

42. H. Isliker, L. Vlahos, A.O. Benz, A. Raoult: Astr. Astroph., 336, 371 (1998) 